\title{
Enhanced Pyroelectric Linear Arrays for Infrared Spectroscopy
}

\author{
Reinhard Köhler1, Dimitar Wassilew', Volkmar Norkus², Marco Schossig², Günter Hofmann ${ }^{1}$ \\ 1) DIAS Infrared GmbH, Pforzheimer Str. 21, 01189 Dresden, Germany \\ 2) Technische Universität Dresden, Institute of Solid State Electronics, 01062 Dresden, Germany \\ r.koehler@dias-infrared.de
}

\begin{abstract}
:
Pyroelectric linear arrays can be used in dispersive sensor systems for analysis of chemical substances. Due to their outstanding properties, such as high signal-to-noise-ratio, low drift effects and a wide spectral range of sensitivity, lithium tantalate sensors PYROSENS, manufactured by DIAS Infrared $\mathrm{GmbH}$, are particularly suitable. In the last several years, design and manufacturing technology of these linear arrays has been improved significantly to meet the demands of spectroscopy even better. At constant element pitch $(100 \mu \mathrm{m}, 50 \mu \mathrm{m}$ or $25 \mu \mathrm{m})$, the pixel length has been increased (typically to 500 $\mu \mathrm{m})$ and the noise equivalent power (NEP) has been reduced by minimizing the chip thickness down to $5 \mu \mathrm{m}$ by means of ion beam etching. Applying and structuring an additional highly absorbing nickelchromium layer onto the linear array sensor surface, further potential for optimization exists through the increase and homogenization of spectral absorptance in the sensor pixels. By using new highly specialized ion beam etching technology, the thermal crosstalk has been reduced by introducing a few micrometer narrow separation trenches between the pixels. In such a way, the sensors can be modulated at $10 \mathrm{~Hz}$ with respect to the NEP and show a good spatial resolution, however. On the basis of the improved linear arrays, in combination with a linear variable filter very compact and high-resolution dispersive sensor systems can be constructed. These may operate either in a transmission configuration or as an attenuated total reflection system. For demonstration purposes, a transmission system was developed, which shows the enhanced functionality simply.
\end{abstract}

Key words: pyroelectric detector, linear array, absorber, spectroscopy, spectrometer

\section{Introduction}

Linear arrays based on pyroelectric sensors are used in calibrated temperature-measuring systems as well as for infrared spectroscopy. In both cases, they must feature high sensitivity, high signal-to-noise ratio, good spatial resolution and spectral responsivity which is as uniform as possible. For many years, linear pyroelectric arrays based on monocrystalline lithium tantalate have proved their worth. These sensors are hybrid components in a metal hermetic housing with an integrated IR filter window fitting to the respective application. Their typical pixel number is 128 with a pitch of $100 \mu \mathrm{m}$, or 256 with a pitch of $50 \mu \mathrm{m}$. In the meantime, arrays with 510 pixels in a $25 \mu \mathrm{m}$ pitch are also available. The functional scheme of a pyroelectric linear array is shown in Fig. 1 while the mechanical basic structure of a PYROSENS array with 256 pixels is shown in Fig. 2. Usually, the $\mathrm{LiTaO}_{3}$ sensor chip and the readout circuit are placed side by side on a common support beam. Each pixel is connected by wire bonding to an input amplifier of the readout circuit.

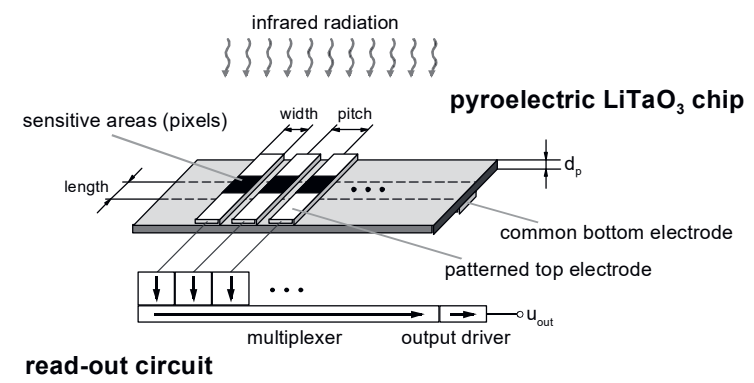

Fig. 1. Functional principle of pyroelectric linear array 


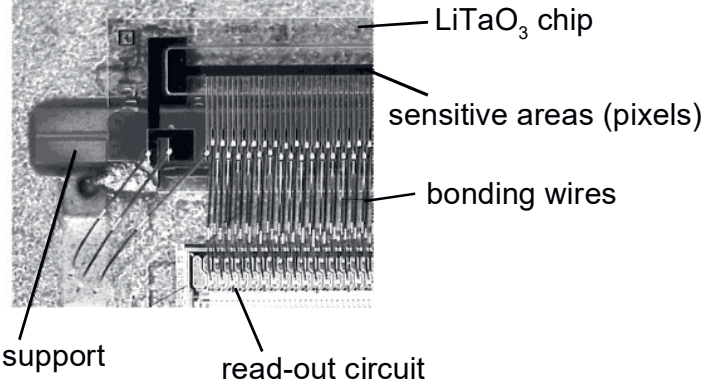

Fig. 2. Detail of a 256 pixel linear array PYROSENS with $\mathrm{LiTaO}_{3}$ sensor chip

Pyroelectric sensor chips generate an output signal when their intrinsic temperature changes. This temperature change is realized by absorption of the infrared radiation, which is to be characterized. Since the pyroelectric detector is an AC-detector, the measured radiation has to be modulated.

\section{Thin Sensing Elements}

The simplified formula (1) applies in current mode operated pyroelectric sensors for modulation frequency above $10 \mathrm{~Hz}$.

$$
\begin{array}{ll}
R= & \frac{\tau_{w} \alpha p v}{C_{P} d_{p} 2 C f_{c h}} \\
R & \text { responsivity } \\
\tau_{W} & \text { transmittance of the optical window } \\
\alpha & \text { absorptance of the responsive element } \\
p & \text { pyroelectric coefficient } \\
V & \text { amplification factor } \\
C_{P} & \text { volume-specific heat of the pyroelectric } \\
& \text { material } \\
d_{P} & \text { thickness of sensitive element } \\
C & \text { integration capacity } \\
f_{C h} & \text { chopping frequency }
\end{array}
$$

The responsivity $R$ of a pyroelectric sensor can be increased significantly by reducing the thickness of the pyroelectric sensor chip. Considering the signal-to-noise ratio, for which the specific detectivity $D^{*}$ of a sensor is a common measure, the behavior shown in Fig. 3 results for a single sensor element.

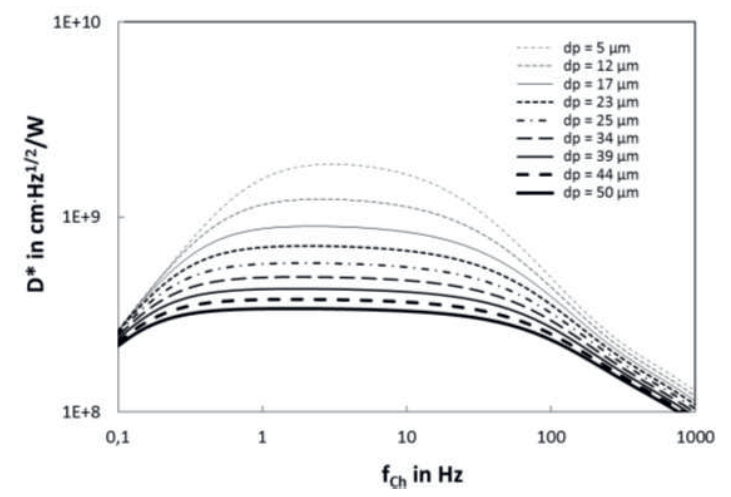

Fig. 3. Effect of the sensor thickness on specific detectivity $D^{*}$ (sensing area $2 \times 2 \mathrm{~mm}^{2}$ )
By using ion beam etching technology it is possible to reduce sensor chip thickness down to approx. $5 \mu \mathrm{m}$. In order to ensure the mechanical stability and stiffness of the linear array, only the area near the sensor elements is etched, while a stabilizing frame with a thickness of approx. $25 \mu \mathrm{m}$ is left on the outside. Although the vacuum etching process is very complex and takes a long time, the effort is certainly worthwhile, since it results in a significantly improved sensitivity and signal-to-noise ratio.

\section{Thermally Insulating Trenches}

In the case of multi-element sensors, such as linear arrays with closely adjacent sensor elements (pixels), the spatial resolution is also important in addition to the sensitivity. For this purpose, the best possible thermal separation of the individual pixels is to be applied, which keeps the heat flux in neighboring pixels as low as possible.

The pyroelectric $\mathrm{LiTaO}_{3}$ sensors PYROSENS have the optimum signal-to-noise ratio typically between $5 \mathrm{~Hz}$ and $10 \mathrm{~Hz}$ modulation frequency. However, because of the high thermal crosstalk in unstructured sensor chips with a pixel spacing of 5-10 $\mu \mathrm{m}$ (depending on the type), modulation frequencies below $80 \mathrm{~Hz}$ are generally not useful. Particularly for spectroscopic applications in which low modulation frequencies would be possible in principle, a better thermal insulabetween the pixels would open up new fields of application.

A high-performance ion beam etching system has been available for micro-structuring since 2014 , offering new technological possibilities in this field. The corresponding work was carried out on both 128-element and 256-element line sensors. According to the design of geometrically improved trench structures, the ion beam etching parameters were set in extensive technological tests in such a way that optimal thermally insulating trenches can be produced with high yield.

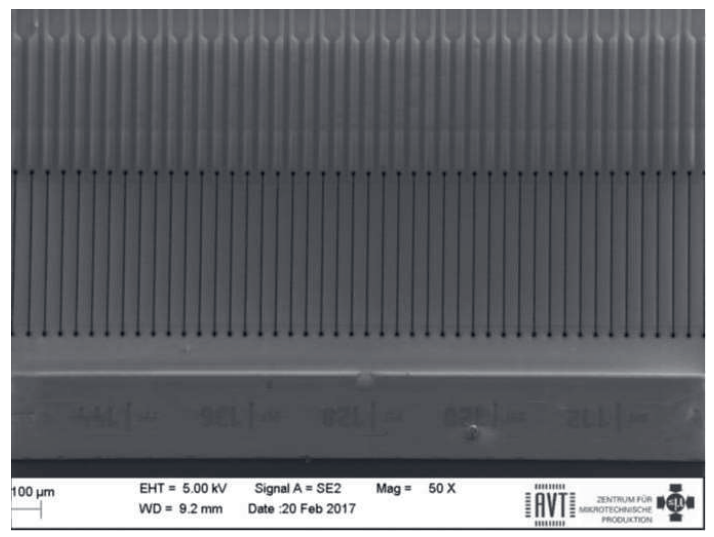

Fig. 4. SEM picture of insulation trenches in a 256 pixel linear array (pixel length $500 \mu \mathrm{m}$, pitch $50 \mu \mathrm{m}$ ) 
Optimal separation is achieved when the thin lithium tantalate chip is completely etched through, but the trench is not wider than the distance between the electrodes of the individual sensor elements at its widest point. For 128element sensors $10 \mu \mathrm{m}$ are available and for 256-element sensors $8 \mu \mathrm{m}$ only.

Fig. 4 shows a scanning electron micrograph of a linear array structured with this technology. The $\mathrm{LiTaO}_{3}$ sensor chip is approximately $5 \mu \mathrm{m}$ thick in the region of the radiation-sensitive pixels. The image shows a section of a 256 element array with $500 \mu \mathrm{m}$ long pixels in a grid of $50 \mu \mathrm{m}$. The etched structure is very uniform. Fig. 5 demonstrates the high quality of the ion beam etched trenches once again in a higher magnification. The round shape of the trench end was chosen to reduce the risk of stress cracking at the trench runout. For the actual trench, the measurement results in a maximum width on the upper side of about $5.2 \mu \mathrm{m}$ and a minimum width on the bottom side of about $0.6 \mu \mathrm{m}$

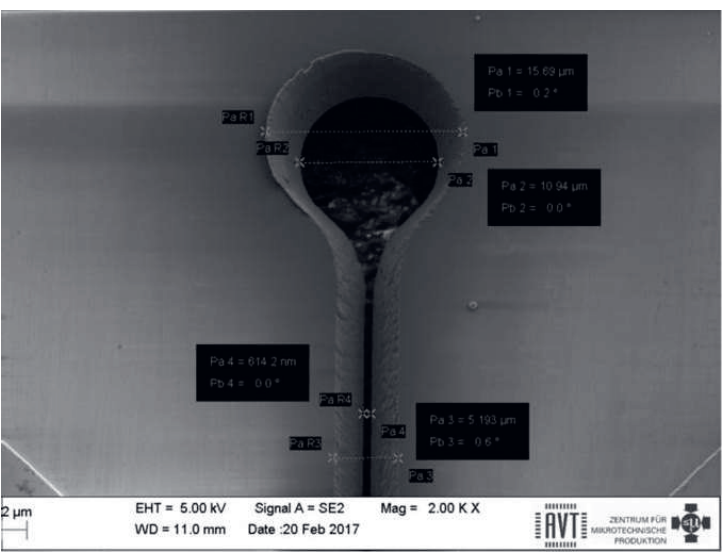

Fig. 5. Detail of an insulation trench in a 256 pixel linear array (SEM magnification 2000)

The main results of comprehensive measurements on two 256-element $\mathrm{LiTaO}_{3}$ sensor arrays type PYROSENS 256LTI SL (with insulation trenches) are shown in Fig. 6 and 7 and are compared there with the measured values of a PYROSENS 256LTI type sensor without trenches but with identical element geometry. The representation of the frequency response of noise-equivalent power (NEP) as a measure of signal-to-noise ratio of one pixel shows that this is higher (lower NEP) at a lower modulation frequency. In the case of the NEP, no significant differences could be found between the arrays with and without etched trenches. A reduction of the NEP by the introduction of the trenches cannot be observed. The deviations between the three measured curves are due to the technologically caused slight differences in the sensor chip thickness.

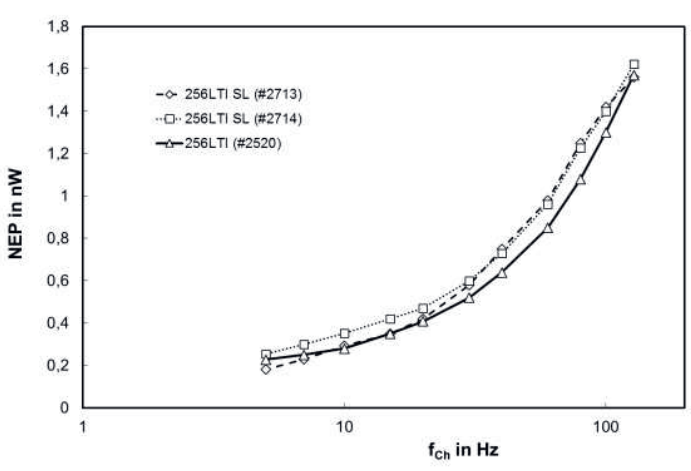

Fig. 6. Noise equivalent power NEP vs. modulation frequency for 256 pixel linear arrays with trenches (256LTI SL) and w/o trenches (256LTI) (measured @ $25^{\circ} \mathrm{C}$ with $400 \mathrm{~K}$ black body)

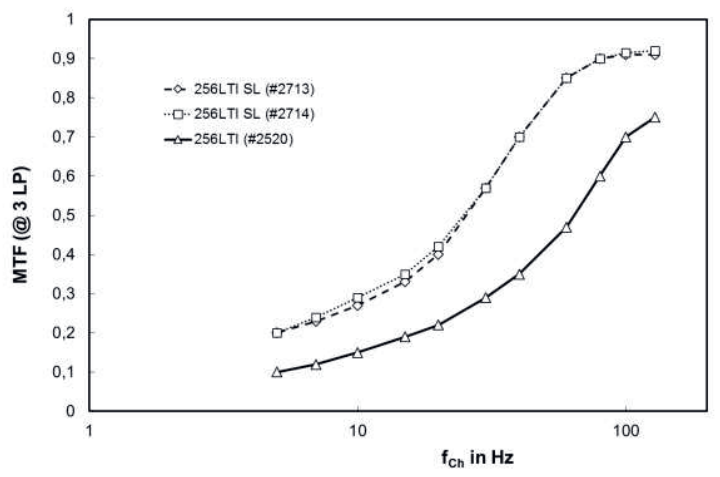

Fig. 7. Modulation transfer function MTF @ 3 line pairs vs. modulation frequency for 256 pixel linear arrays with trenches (256LTI SL) and w/o trenches (256LTI) (measured @ $25^{\circ} \mathrm{C}$ with $400 \mathrm{~K}$ black body)

Fig. 7 shows the frequency response of the Modulation Transfer Function (MTF) for 3 line pairs (LP) of the same sensors as a measure of the spatial resolution. As expected, the improvement of the MTF by reducing the thermal crosstalk in the sensors with ion beam etched trenches between the pixels is well recognizable over the entire measured modulation frequency range between $5 \mathrm{~Hz}$ and $128 \mathrm{~Hz}$.

\section{Metal Thin Film Absorber}

In order to achieve a better, and, above all, spectrally uniform, radiation absorption, two types of absorbers are commonly used in singleelement sensors of DIAS Infrared $\mathrm{GmbH}$. Unfortunately, both absorbers cannot be used for linear arrays because of technological incompatibilities. This is, on the one hand, a silver black coating with an excellent absorption of more than $98 \%$ in a wide spectral range, which can be structured only very roughly by means of metal shadow masks. The patterning accuracy is worse than $100 \mu \mathrm{m}$ and thus, larger than the width of a pixel of a line sensor. On the other hand, a polymer absorber is used, but the 
coating technology makes it impossible to apply it on the linear arrays.

Therefore only linear arrays without additional absorber have been produced until now. These linear arrays feature absorption-optimized metal electrodes, so that a large part of the IR radiation is absorbed in the sensor material itself. However, the spectral absorption is not uniform and features interferences which results in a wavelength- and thickness-dependent responsivity. This is a drawback for spectroscopic applications. But if an additional thin-film absorber can be integrated on the $5 \mu \mathrm{m}$ thick, 3D microstructured pixels, the $\mathrm{LiTaO}_{3}$ linear arrays would reach a new quality level.

At the Dresden University of Technology, a nanostructured metal thin-film absorber on the basis of a nickel chromium ( $\mathrm{NiCr}$ ) alloy was developed that meets the following requirements:

$-\alpha \geq 0.9$

- thickness $\leq 1 \mu \mathrm{m}$

- can be structured photolithographically

- low heat capacity

- good heat conduction into sensor element

- technology compliant

- low process temperature $\left(<120^{\circ} \mathrm{C}\right)$

- long-term stable and reproducible

- cost-effective.

It is a very promising approach for industrial use in the manufacture of thermal infrared sensors in mass production. The patented process has been optimized, so that it can be used to produce nanostructured thin-film absorption layers on PYROSENS linear arrays.

The so-called GLAD technology (glancing angle deposition) is used for the generation of these absorption layers. The principle is illustrated in Fig. 8 [1].

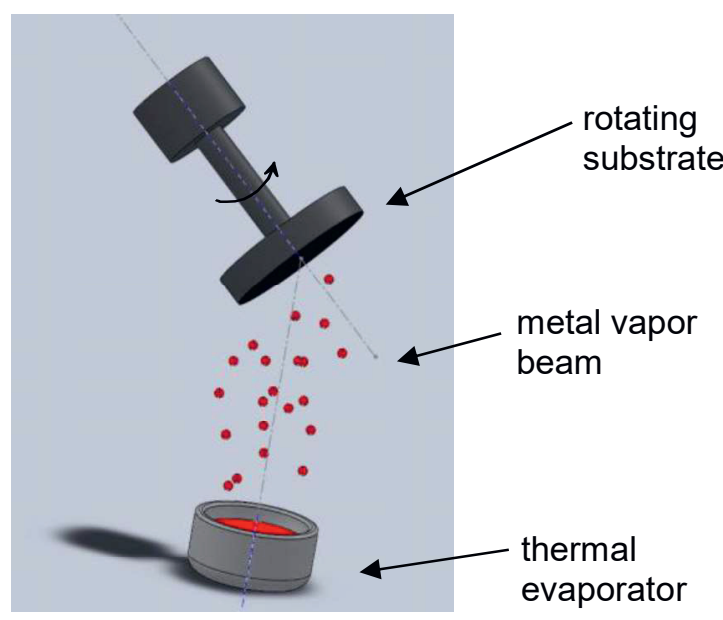

Fig. 8. Schematic arrangement for the GLAD deposition of NiCr thin-film absorption layers
The substrate is rotating and the vapor particles arrive at a very small angle $\left(1^{\circ}\right.$ to $\left.5^{\circ}\right)$ to the evaporator axis. Due to atomic shadowing effects, the vapor particles deposit at preferred condensation points and a column-shaped layer structure is formed. Fig. 9 shows a SEM micrograph of such a deposited NiCr layer.

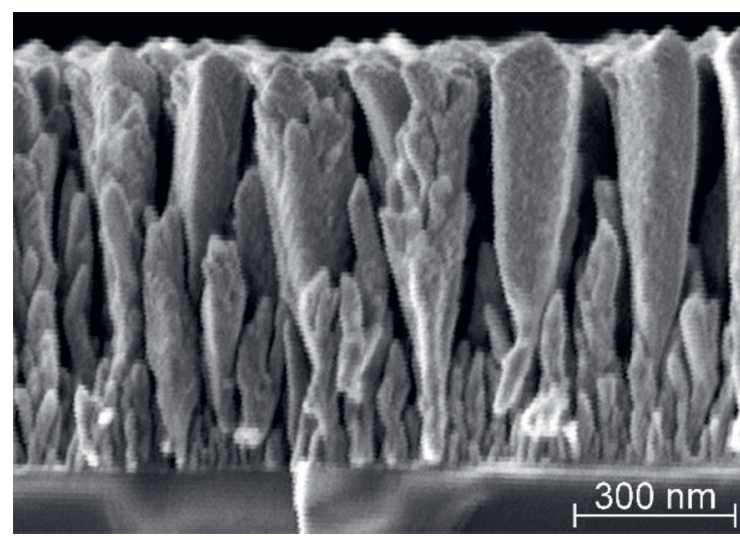

Fig. 9. Section of $\mathrm{NiCr}$ thin-film absorber

Due to the porous structure, absorption coefficients higher than 0.85 are achieved even with a layer thickness below $1 \mu \mathrm{m}$. Depending on the process parameters, absorptivity can be optimized for different substrates and wavelength ranges.

The metal thin-film absorber can be structured photolithographically. A subtraction etching method has been tested as well as a lift-off process. Fig. 10 shows the absorber on 256 pixel linear arrays with a $100 \mu \mathrm{m}$ by $42 \mu \mathrm{m}$ area of each pixel, structured with both methods.

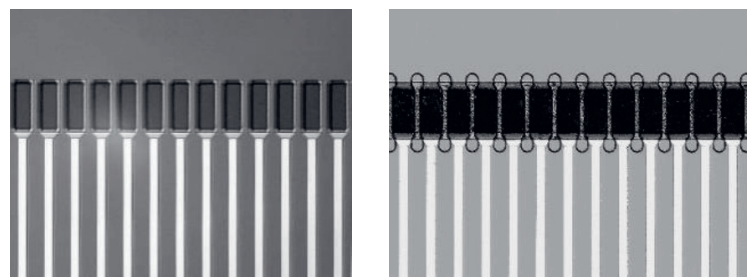

Fig. 10. NiCr thin film absorber on $100 \times 42 \mu m^{2}$ sized pixels of $\mathrm{LiTaO}_{3}$ linear arrays, patterned by lift-off (left) and by etching (right)

The absorption characteristic of $\mathrm{LiTaO}_{3}$ sensors without absorber and with silver black coating are compared to those of sensors with the new $\mathrm{NiCr}$ absorber (Fig. 11).

In the case of the sensor without absorber on top of the lithium tantalate chip ( $25 \mu \mathrm{m}$ thick), the strong periodic variation of the absorption caused by interferences is clearly visible. The newly developed $\mathrm{NiCr}$ absorber is very close to the very good absorptance $\alpha$ of the silver black coating. 


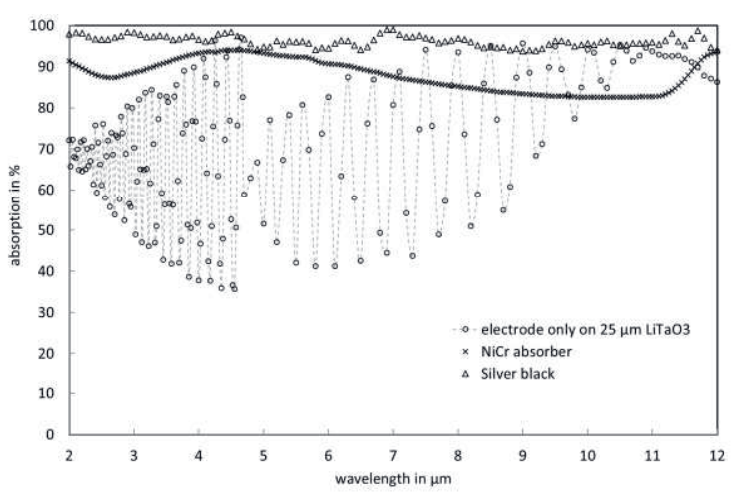

Fig. 11. Absorption behavior of different sensor top layers

Finally, the opportunity to produce $\mathrm{LiTaO}_{3}$ sensor chips with very thin sensing elements, separated by thermally insulating trenches and coated with a high absorptance $\mathrm{NiCr}$ thin-film absorber leads to pyroelectric linear arrays with high sensitivity and outstanding signal-to-noise, which are homogeneous over a wide spectral range from $2 \mu \mathrm{m}$ to $20 \mu \mathrm{m}$. Such linear arrays are ideal for the use in spectroscopy.

\section{Spectrometer Demonstrator}

On the basis of linear arrays equipped with additional absorbers and separation trenches in combination with a linear variable filter (LVF), very compact and high-resolution dispersive sensor systems can be constructed. These may operate either in a transmission configuration or as an attenuated total reflection (ATR) system (Fig. 12, 13).

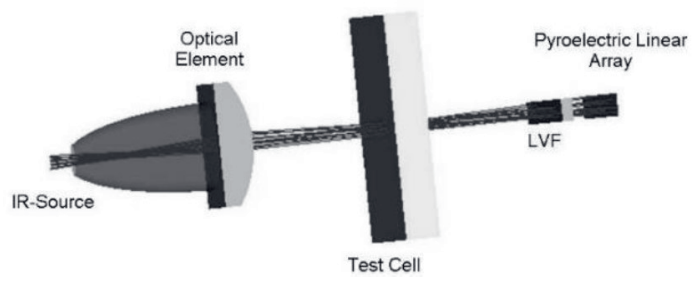

Fig. 12. Dispersive spectroscopy system in transmission configuration

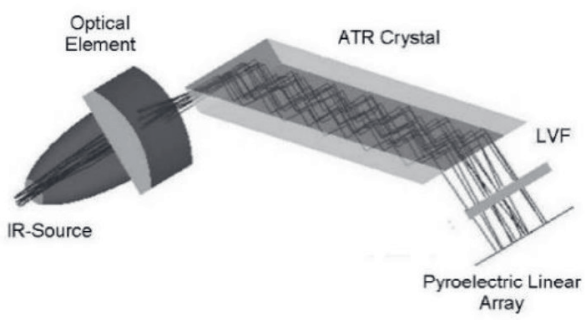

Fig. 13. Dispersive spectroscopy system in ATR configuration

In order to show the potential of the described linear arrays of the type PYROSENS and the comparatively easy use, a spectrometer module was constructed. The compact and portable spectrometer shown in Fig. 14 operates in the transmission mode, but can be transformed to an ATR spectrometer as well. It consists essentially of a black body IR source, a chopper assembly, a sample holder and the linear array. A linear variable filter (LVF) is mounted on the array so that each pixel is sensitive to a different wavelength. The sensor is interchangeable so that linear arrays with different LVFs or other IR filters for different wavelength ranges can be tested.

The complete control and synchronization of all parts is realized by means of an evaluation kit, which is offered by DIAS Infrared $\mathrm{GmbH}$ as standard for the control of the linear arrays of the type PYROSENS. This small PCB is handling the output signal of the linear array and transfers it via a USB interface to a computer.

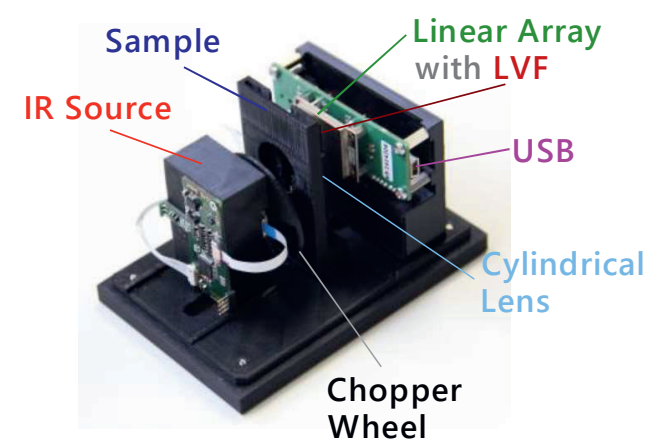

Fig. 14. Compact spectrometer module with pyroelectric linear array with linear variable filter (LVF)

To show the good results even without any mathematical signal manipulation (except zeropoint correction) a PTFE sample has been characterized, as it is provided for testing commercial FTIR spectrometers. The results are shown in Fig 15. For comparison, the transmission spectrum of the sample has been measured with a PerkinElmer 'Frontier' FTIR spectrometer. In the module two linear arrays PYROLINE have been used, one with a LVF covering $3 \mu \mathrm{m}$ to $5 \mu \mathrm{m}$ and another one with a LVF covering the range from $5.5 \mu \mathrm{m}$ to $11 \mu \mathrm{m}$.

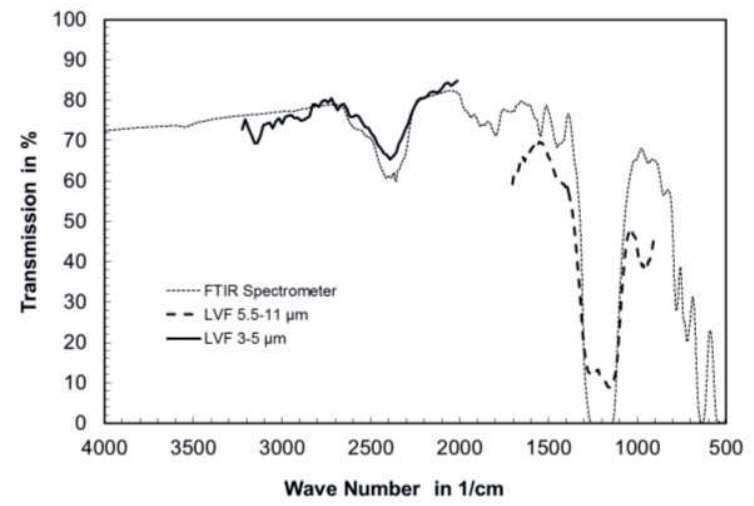

Fig. 15. Transmission spectrum of a PTFE test sample measured with the spectrometer module and a PerkinElmer FTIR spectrometer 
The work has been funded by the Saxon State Ministry of Economic Affairs, Labor and Transport and the European Union under the code 100244480.

\section{References}

[1] M. Schossig, V. Norkus, G. Gerlach, Broadband nickel-chromium thin-film absorber for thermal sensors, Eurosensors XXII, Dresden, Germany, 7-10 September 2008. Proceedings. Düsseldorf: VDI 2008. 873-876. (on CD-ROM ISBN 978-3$00-025217-4$ ) 